\title{
Article \\ Clinical Characteristics and Relevance of Oral Candida Biofilm in Tongue Smears
}

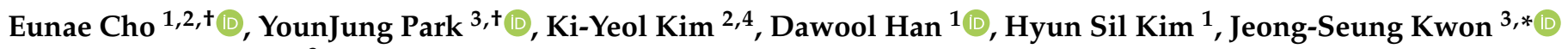 \\ and Hyung-Joon Ahn ${ }^{3, *}$ \\ 1 Department of Oral Pathology, Oral Cancer Research Institute, Yonsei University College of Dentistry, \\ Seoul 03722, Korea; sandra@yuhs.ac (E.C.); ipodvideo@yuhs.ac (D.H.); KHS@yuhs.ac (H.S.K.) \\ 2 BK21 FOUR Project, Yonsei University College of Dentistry, Seoul 03722, Korea; KKY1004@yuhs.ac \\ 3 Department of Orofacial Pain and Oral Medicine, Dental Hospital, Yonsei University College of Dentistry, \\ Seoul 03722, Korea; DARKSTAR@yuhs.ac \\ 4 Department of Dental Education, Yonsei University College of Dentistry, Seoul 03722, Korea \\ * Correspondence: JSKWON@yuhs.ac (J.-S.K.); HJAHN@yuhs.ac (H.-J.A.); \\ Tel.: +82-2-2228-3111 (J.-S.K.); +82-2-2228-3112 (H.-J.A.) \\ + Equally contributed to this article.
}

check for updates

Citation: Cho, E.; Park, Y.; Kim, K.-Y.; Han, D.; Kim, H.S.; Kwon, J.-S.; Ahn, H.-J. Clinical Characteristics and Relevance of Oral Candida Biofilm in Tongue Smears. J. Fungi 2021, 7, 77. https://doi.org/10.3390/jof7020077

Received: 22 December 2020

Accepted: 20 January 2021

Published: 22 January 2021

Publisher's Note: MDPI stays neutral with regard to jurisdictional claims in published maps and institutional affiliations.

Copyright: (c) 2021 by the authors. Licensee MDPI, Basel, Switzerland. This article is an open access article distributed under the terms and conditions of the Creative Commons Attribution (CC BY) license (https:// creativecommons.org/licenses/by/ $4.0 /)$.
Abstract: Dimorphic Candida exist as commensal yeast carriages or infiltrate hyphae in the oral cavity. Here, we investigated the clinical relevance of Candida hyphae in non-pseudomembranous oral candidiasis (OC) by smears of tongue biofilms. We conducted a retrospective study of 2829 patients who had had tongue smears regardless of OC suspicion. Clinical characteristics were evaluated using a novel method of assessing hyphae. Clinical factors (moderate/severe stimulated pain, pain aggravated by stimulation, tongue dorsum appearance and initial topical antifungal use) were highly significant in the high-grade hyphae group but were statistically similar in the low-grade hyphae and non-observed hyphae group, suggesting low-grade hyphae infection as a subclinical OC state. In addition to erythematous candidiasis (EC), a new subtype named "morphologically normal symptomatic candidiasis" (MNSC) with specific pain patterns and normal tongue morphology was identified. MNSC had a significantly higher proportion of moderate and severe stimulated pain cases than EC. Low unstimulated salivary flow rate $(<0.1 \mathrm{~mL} / \mathrm{min})$ was found to be a common risk factor in MNSC and EC. In non-pseudomembranous OC, pain patterns were dependent on Candida hyphae degree regardless of tongue dorsum morphology. Morphologic differences seen in high-grade hyphae infection were not associated with systemic diseases or nutritional deficiencies.

Keywords: oral candidiasis; Candida; hyphae; biofilm; erythematous candidiasis; atrophic candidiasis; smears; tongue

\section{Introduction}

Candida are dimorphic fungus presented as commensal yeast carriages or invasive hyphae [1]. The hyphae phenotype is associated with virulence, epithelial infiltration, tissue damage, keratinization and biofilm formation [2-8]. Thus, hyphae identification is necessary in detecting oral candidiasis (OC) transition from commensal Candida [9]. Recently, the biofilm formation is assumed to have a critical role in OC in addition to hyphae transition [10-12]. Candida biofilm comprises a sessile-shaped heterogenous ecosystem, which includes Candida microorganisms, extracellular matrix and sometimes bacteria [13]. Compared to planktonic Candida, biofilm formation induces persistent infection, recurrence and antifungal resistance and is essential for Candida pathogenicity in mucosal candidiasis [12-15].

OC is typically classified according to its clinical manifestations, representatively, pseudomembranous candidiasis (PC) and erythematous candidiasis (EC) [16,17]. In the oral cavity, the tongue is the primary reservoir for Candida colonization [18]. Candida 
biofilms are assumed to be causative for PC in the soft tissue, including the tongue $[10,19]$. However, there is no known association between Candida biofilms and non-PC subtypes. Importantly, it is unclear what clinical manifestations, aside from whitish curd-like material (pseudomembrane), are relevant to Candida biofilms. In this study, we assessed the tongue biofilm to evaluate the relevance of clinical characteristics and Candida hyphae in nonPC subtypes.

\section{Materials and Methods}

\subsection{Data Collection, Study Design and Variable Definition}

This retrospective study, approved by the Institutional Review Board at Yonsei University Dental Hospital (2-2017-0001), was based on medical records and smear slides of patients that had visited Yonsei University Dental Hospital from 2014 to 2019 and had received a tongue smear $(n=2829)$. Clinically confirmed cases of oral PC were later excluded during the study.

Assessment factors were age, sex, pain characteristics (spontaneous pain [SpP], stimulated pain [StP] and pain difference by stimulation [PDSt]), tongue dorsum morphology, coexisting oral and systemic conditions, pathologic hyphae grade, salivary flow rate, complete blood counts and nutrition blood levels. The criteria of assessment factors are specified in Table 1. Pain characteristics had been evaluated by subjective pain response based on the numerical rating scale (NRS). Ulcerative or erosive oral diseases that could mask tongue pain were excluded during pain record evaluation. Tongue dorsum morphology was assessed by medical records and (if available) previous clinical photos.

Table 1. Criteria of clinical and pathologic factors.

\begin{tabular}{|c|c|}
\hline Factors & Criteria \\
\hline \multicolumn{2}{|l|}{ Pain characteristics } \\
\hline Spontaneous pain $(\mathrm{SpP})$ & Intensity: 0-10 (NRS) \\
\hline Stimulated pain (StP) & Intensity: 0-10 (NRS) \\
\hline Pain intensity & Mild, $<$ NRS4; moderate, $\leq$ NRS4, $<$ NRS7; severe, $7 \leq$ NRS $\leq 10$ \\
\hline \multirow{3}{*}{$\begin{array}{l}\text { Pain difference by stimulation } \\
\quad \text { (StP-SpP intensity) }\end{array}$} & Positive value: pain aggravation by stimulation (AggSt) \\
\hline & $0:$ no pain difference \\
\hline & Negative value: pain alleviation by stimulation (AllSt) \\
\hline \multirow[b]{2}{*}{ Tongue dorsum morphology } & Atrophy: any partial or full tongue papilla atrophy \\
\hline & $\begin{array}{l}\text { Normal: a pink mucosa with normal tongue papilla or mild keratotic } \\
\text { tongue papilla/mucosa }\end{array}$ \\
\hline \multirow{4}{*}{ Hyphae grade ${ }^{\text {a }}$} & High-grade hyphae (HGH): hyphae value $\geq 10$ \\
\hline & Low-grade hyphae (LGH): hyphae value $<10$ \\
\hline & No observed hyphae (NH): no hyphae \\
\hline & $\begin{array}{l}\text { Insufficient material: smear slides with fewer than ten high-power } \\
\text { fields of valid oral material }\end{array}$ \\
\hline
\end{tabular}

Abbreviations: NRS, numeric rating scale. ${ }^{\text {a }}$ Based on the total sum of Candida hyphae in ten selected high-power fields in the order of highest hyphae aggregations on smear slides.

Tongue biofilms had been sampled from the dorsal tongue with a wooden tongue depressor and smeared onto glass slides. The slides, which had been fixed with ethyl alcohol and stained with Periodic acid-Schiff (PAS) stain, were put under a light microscope for pathologic examination of Candida hyphae.

Candida hyphae were evaluated using a novel method established by our group. Hyphae were first divided into the existence of hyphae $(\mathrm{H})$ group and the no observed hyphae (NH) group. The H group was further designated either low-grade hyphae (LGH) or high-grade hyphae (HGH) that was divided by a hyphae value cut-off point selected based on a preliminary study described in Figure S1 and Table S1. We analyzed clinical pain and morphological characteristics with the total Candida hyphae value in 48 smeared slides 
to identify specific correlation patterns. The clinical characteristics did not present a linear correlation pattern with the total hyphae count, but we noticed that certain characteristics, such as StP intensity and PDSt, revealed specific patterns in the range of hyphae values over a cut-off point (Figure S1). Next, we analyzed candidates of the hyphae value cut-off point within the range of 6 to 50, to determine an accurate cut-off point that had the most sensitivity and specificity for clinical relevance-being 10 (Table S1). Based on the cut-off point 10, LGH and HGH were defined as detailed in Table 1. Since total hyphae counts equal and over 10 were uniformly classified as HGH, the total sum of Candida hyphae at ten selected high-power fields in the order of highest hyphae aggregations were used for hyphae grade assessment in the final analysis.

Due to the severe imbalance between the NH group and the $\mathrm{H}(n=135) / \mathrm{HGH}$ $(n=69) / \mathrm{LGH}(n=66)$ groups, we randomly selected control cases $(n=205)$ from the NH group within 3 folds of the hyphae group of interest (HGH).

\subsection{Statistics}

The distribution and proportion of the data based on pathologic groups were analyzed by Mann-Whitney U test, Kruskal-Wallis $\mathrm{H}$ test and Chi-square test based on the characteristics of the dataset. Hyphae value cut-off points were analyzed in terms of sensitivity, specificity and accuracy. Risk factors were evaluated by simple and multiple logistic regression (odds ratio [OR] and 95\% confidence interval [CI]), and the model was evaluated using receiver operating characteristic (ROC) curve analysis. $p<0.05$ were considered significant. Statistics were analyzed with IBM SPSS Statistics for Windows, Version 25.0. Armonk, NY: IBM Corp.

\section{Results}

\subsection{Basic Characteristics of the Study}

Tongue smears from a total of 2829 patients were primarily included for evaluation. The smears had been routinely conducted in patients with a wide range of chief complaints in the oral soft tissue, regardless of OC suspicion or tongue pain. Chief complaints included but were not limited to oral pain, painless benign or malignant soft tissue lesions, trauma, dry mouth, halitosis or neuropathic disorder in the overall oral cavity.

Among them, Candida hyphae were observed in the smears of 244 patients (8.6\%). Twenty-three cases yielded insufficient smear material and were excluded. Eight of the 244 patients were confirmed PC and, thus, excluded.

The final dataset $(n=340)$, after exclusion of inappropriate data and random selection, had a median age of 64 years (interquartile range [IQR], 55-73), 79.7\% being female (Table 2).

Table 2. Clinical characteristics based on Candida hyphae grade.

\begin{tabular}{|c|c|c|c|c|c|}
\hline \multirow{2}{*}{ Characteristics } & \multirow{2}{*}{ Entire Study } & \multicolumn{2}{|c|}{$\mathbf{H}$} & \multirow{2}{*}{ NH } & \multirow{2}{*}{$\begin{array}{c}p \text { Value } \\
\text { H/NH } \\
\text { HGH/LGH/NH }\end{array}$} \\
\hline & & HGH & LGH & & \\
\hline \multicolumn{6}{|c|}{ Number of subjects/total (\%), unless otherwise stated } \\
\hline \multicolumn{6}{|c|}{ Age } \\
\hline \multirow{2}{*}{ Median (IQR), years } & \multirow{2}{*}{$64(55-73)$} & \multicolumn{2}{|c|}{$68(60-77)$} & \multirow{2}{*}{$61(53-70)$} & $<0.001$ \\
\hline & & $70(61-76)$ & $67(58-77)$ & & $<0.001$ \\
\hline \multirow{2}{*}{$<49$ years } & \multirow{2}{*}{$55 / 340(16.2)$} & \multicolumn{2}{|c|}{$15 / 135(11.1)$} & \multirow{2}{*}{$40 / 205$ (19.5) } & $<0.001$ \\
\hline & & $7 / 69(10.1)$ & $8 / 66(12.1)$ & & $<0.001$ \\
\hline \multirow{2}{*}{$50-69$ years } & \multirow{2}{*}{$174 / 340(51.2)$} & $58 / 1$ & 3.0) & \multirow{2}{*}{$116 / 205(56.6)$} & \\
\hline & & $27 / 69(39.1)$ & $31 / 66(47.0)$ & & \\
\hline \multirow{2}{*}{$\leq 70$ years } & \multirow{2}{*}{$111 / 340(32.6)$} & $62 / 1$ & 5.9) & \multirow{2}{*}{$49 / 205(23.9)$} & \\
\hline & & $35 / 69(50.7)$ & $27 / 66(40.9)$ & & \\
\hline \multirow{3}{*}{ Female (\%) } & \multicolumn{3}{|c|}{ Sex } & & \\
\hline & \multirow{2}{*}{27} & $112 /$ & 33.0) & \multirow{2}{*}{$159 / 205(77.6)$} & 0.226 \\
\hline & & $58 / 69(84.1)$ & $54 / 66(81.8)$ & & 0.455 \\
\hline
\end{tabular}


Table 2. Cont.

\begin{tabular}{|c|c|c|c|c|c|}
\hline \multirow{2}{*}{ Characteristics } & \multirow{2}{*}{ Entire Study } & \multicolumn{2}{|c|}{$\mathbf{H}$} & \multirow{2}{*}{ NH } & \multirow{2}{*}{$\begin{array}{c}p \text { Value } \\
\text { H/NH } \\
\text { HGH/LGH/NH }\end{array}$} \\
\hline & & HGH & LGH & & \\
\hline \multicolumn{6}{|c|}{ Pain symptoms } \\
\hline \multirow{2}{*}{ Presence of SpP } & \multirow{2}{*}{$174 / 340(51.2)$} & \multicolumn{2}{|c|}{ 69/135 (51.1) } & \multirow{2}{*}{$105 / 205(51.2)$} & 0.984 \\
\hline & & $38 / 69(55.1)$ & $31 / 66(47.0)$ & & 0.642 \\
\hline \multirow{2}{*}{$\begin{array}{l}\text { SpP intensity, median } \\
\text { (IQR), NRS }\end{array}$} & \multirow{2}{*}{$1(0-4)$} & \multicolumn{2}{|c|}{$2(0-4)$} & \multirow{2}{*}{$1(0-4)$} & 0.831 \\
\hline & & $2(0-5)$ & $0(0-4)$ & & 0.520 \\
\hline \multirow{2}{*}{ Painless } & \multirow{2}{*}{$166 / 340(48.8)$} & \multicolumn{2}{|c|}{ 66/135 (48.9) } & \multirow{2}{*}{$100 / 205(48.8)$} & 0.584 \\
\hline & & \multicolumn{2}{|c|}{$23 / 135(17.0)$} & & 0.728 \\
\hline Mild $^{a}$ & $55 / 340(16.2)$ & $11 / 69(15.9)$ & $12 / 66(18.2)$ & $32 / 205(15.6)$ & \\
\hline \multirow{2}{*}{ Moderate ${ }^{a}$} & \multirow{2}{*}{$93 / 340(27.4)$} & $33 / 1$ & 4.4) & $60 / 205(29.3)$ & \\
\hline & & $\begin{array}{r}20 / 69(29.0) \\
13 /\end{array}$ & $\begin{array}{l}13 / 66(19.7) \\
9.6)\end{array}$ & & \\
\hline Severe “ & $26 / 340(7.6)$ & $7 / 69(10.1)$ & $6 / 66(9.1)$ & $13 / 205(6.3)$ & \\
\hline Des & $185 / 240(544)$ & $98 / 1$ & $2.6)$ & $87 / 205(42.4)$ & $<0.001$ \\
\hline Presence of StP & $183 / 340(34.4)$ & $64 / 69(92.8)$ & $34 / 66(51.5)$ & & $<0.001$ \\
\hline StP intensity, & $25(0-6.5)$ & & & $0(0-6)$ & $<0.001$ \\
\hline median (IQR), NRS & $2.0(0-0.0)$ & $6(4.5-8)$ & $1.3(0-6.4)$ & & $<0.001$ \\
\hline Dainloce & $155 / 340 / 456)$ & $37 / 1$ & $7.4)$ & $118 / 205(57.6)$ & $<0.001$ \\
\hline Paanless & $155 / 340(45.6)$ & $5 / 69(7.2)$ & $32 / 66(48.5)$ & & $<0.001$ \\
\hline Mild a & $25 / 340(74)$ & $11 / 1$ & 8.1) & $14 / 205(68)$ & \\
\hline Modornto a & $86 / 340(253)$ & $\begin{array}{r}6 / 69(8.7) \\
44 / 1 \\
\end{array}$ & $\begin{array}{l}5 / 66(7.6) \\
2.6)\end{array}$ & $42 / 205(205)$ & \\
\hline Moderate $^{a}$ & $86 / 340(25.3)$ & $\begin{array}{r}28 / 69(40.6) \\
43 / 1\end{array}$ & $\begin{array}{l}16 / 66(24.2) \\
1.9)\end{array}$ & $42 / 205(20.5)$ & \\
\hline Severe ${ }^{a}$ & $74 / 340(21.8)$ & $30 / 69(43.5)$ & $13 / 66(19.7)$ & 31/205 (15.1) & \\
\hline PDSt, median (IQR), & $0(0-25)$ & & & $0(0-2)$ & $<0.001$ \\
\hline NRS & $0(0-2.3)$ & $3(1-5)$ & $0(0-2.5)$ & & $<0.001$ \\
\hline Presence of $\mathrm{AcoSt}$ & $163 / 240(479)$ & $87 / 1$ & $4.4)$ & $76 / 205(37.1)$ & $<0.001$ \\
\hline & & $\begin{array}{r}57 / 69(82.6) \\
12 / 1\end{array}$ & $\begin{array}{l}30 / 66(45.5) \\
8.9)\end{array}$ & $43 / 205(210)$ & $<0.001$ \\
\hline Presence of AllSt & $55 / 340(16.2)$ & $1 / 69(1.4)$ & $11 / 66(16.7)$ & $43 / \angle 00(21.0)$ & \\
\hline No pain difference & $122 / 340(35.9)$ & $36 / 1$ & $6.7)$ & $86 / 205(42.0)$ & \\
\hline & & $11 / 69(15.9)$ & $25 / 66(37.9)$ & & \\
\hline & & Tongue dorsal $\mathrm{n}$ & hology & & \\
\hline Normol & $239 / 340(703)$ & $84 / 1$ & $2.2)$ & $155 / 205(756)$ & 0.008 \\
\hline Normal & $239 / 340(10.3)$ & $\begin{array}{r}36 / 69(52.2) \\
51 / 1\end{array}$ & $\begin{array}{l}48 / 66(72.7) \\
37.8)\end{array}$ & $150 / 200(70.6)$ & 0.001 \\
\hline Atrophy & $101 / 340(29.7)$ & $33 / 69(47.8)$ & $18 / 66(27.3)$ & 50/205 (24.4) & \\
\hline & Initial antifunga & erapy response, & cal fluconazole & up, $2 \mathrm{w}$ & \\
\hline Effective on SnP b & & $36 / 1$ & $2.7)$ & & \\
\hline Efrective on spe & & $19 / 55(34.5)$ & $17 / 55(30.9)$ & & 0.824 \\
\hline Non-effective on $\mathrm{SpP}{ }^{\mathrm{b}}$ & & $\frac{7 / 1}{4 / 55(73)}$ & $3 / 55(5-5)$ & & \\
\hline No SnP & & $67 / 1$ & $0.9)$ & & \\
\hline No Spr & & $32 / 55(58.2)$ & $35 / 55(63.6)$ & & \\
\hline 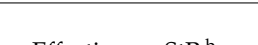 & & $46 / 5$ & 7.4) & & \\
\hline Effective on StP & & $\begin{array}{r}33 / 44(75.0) \\
16 / 4\end{array}$ & $\begin{array}{l}13 / 53(24.5) \\
6.5)\end{array}$ & & $<0.001$ \\
\hline Non-effective on StP ${ }^{b}$ & & $\begin{array}{r}8 / 44(18.2) \\
35 / 5\end{array}$ & $\begin{array}{l}8 / 53(15.1) \\
6.1)\end{array}$ & & \\
\hline No StP & & $3 / 44(6.8)$ & $32 / 53(60.4)$ & & \\
\hline
\end{tabular}

Differences between proportions were determined by the Chi-square test and range distributions were compared by either Mann-Whitney $\mathrm{U}$ test or Kruskal-Wallis $\mathrm{H}$ test. $p$ values $<0.05$ are in bold. ${ }^{a}$ Pain intensity: mild, $<$ NRS4; moderate, $\leq$ NRS4, $<$ NRS7; severe, $7 \leq$ NRS $\leq 10$. ${ }^{\text {b }}$ Effective: full or partial pain relief. Abbreviations: $\mathrm{H}$, existence of hyphae; NH, no observed hyphae; LGH, low-grade hyphae; HGH, high-grade hyphae; IQR, interquartile range; SpP, spontaneous pain; StP, stimulated pain; AggSt, pain aggravation by stimulation; AllSt, pain alleviation by stimulation; NRS, numeric rating scale. 


\subsection{Specific Characteristics of Candida Infection}

Table 2 shows the clinical characteristics of the final dataset based on Candida hyphae grade. The overall percentages of patients showing moderate and severe StP intensity were significantly higher in the HGH group than in the LGH or NH group (40.6 and $43.5 \%$, 24.2 and $19.7 \%, 20.5$ and $15.1 \%$, respectively, $p<0.001$ ). The rate of pain aggravation by stimulation (AggSt) was significantly higher in the HGH group compared to the LGH and $\mathrm{NH}$ groups $(82.6,45.5$ and $37.1 \%$, respectively, $p<0.001)$. Moreover, a higher proportion of pain alleviation by stimulation (AllSt) was seen in the LGH and NH groups (16.7 and 21.0\%, respectively), whereas there was only a single AllSt case (1.4\%) in the HGH group. Clinical characteristics based on hyphae grade revealed that moderate/severe StP intensity and $\mathrm{AggSt}$, but not SpP, were significant in the HGH group; these were assumed to be specific characteristics of non-pseudomembranous subtypes of OC. This was supported by the differences in antifungal response between the hyphae groups. Initial topical fluconazole use for 2 weeks was more significantly effective in the HGH group than the LGH group for StP relief $(p<0.001)$, but not SpP relief $(p=0.502)$. Statistically, however, clinical characteristics had a similar distribution in the LGH and NH groups, implying that the hyphae infection in the LHG group reflected a subclinical OC state.

Among the tongue dorsal morphologic variants, the atrophic appearance was most specific to the HGH group compared to the LGH and NH groups (47.8, 27.3 and $24.4 \%$, respectively, $p<0.01$ ). Despite tongue atrophy being specific to the HGH group, clinically normal tongue morphology predominated in all three hyphae grades, including the HGH group (52.2\%). HGH cases with an atrophic tongue morphology were defined as EC, while painful HGH cases with a normal tongue morphology were defined as "morphologically normal symptomatic candidiasis" (MNSC) (Figure 1).

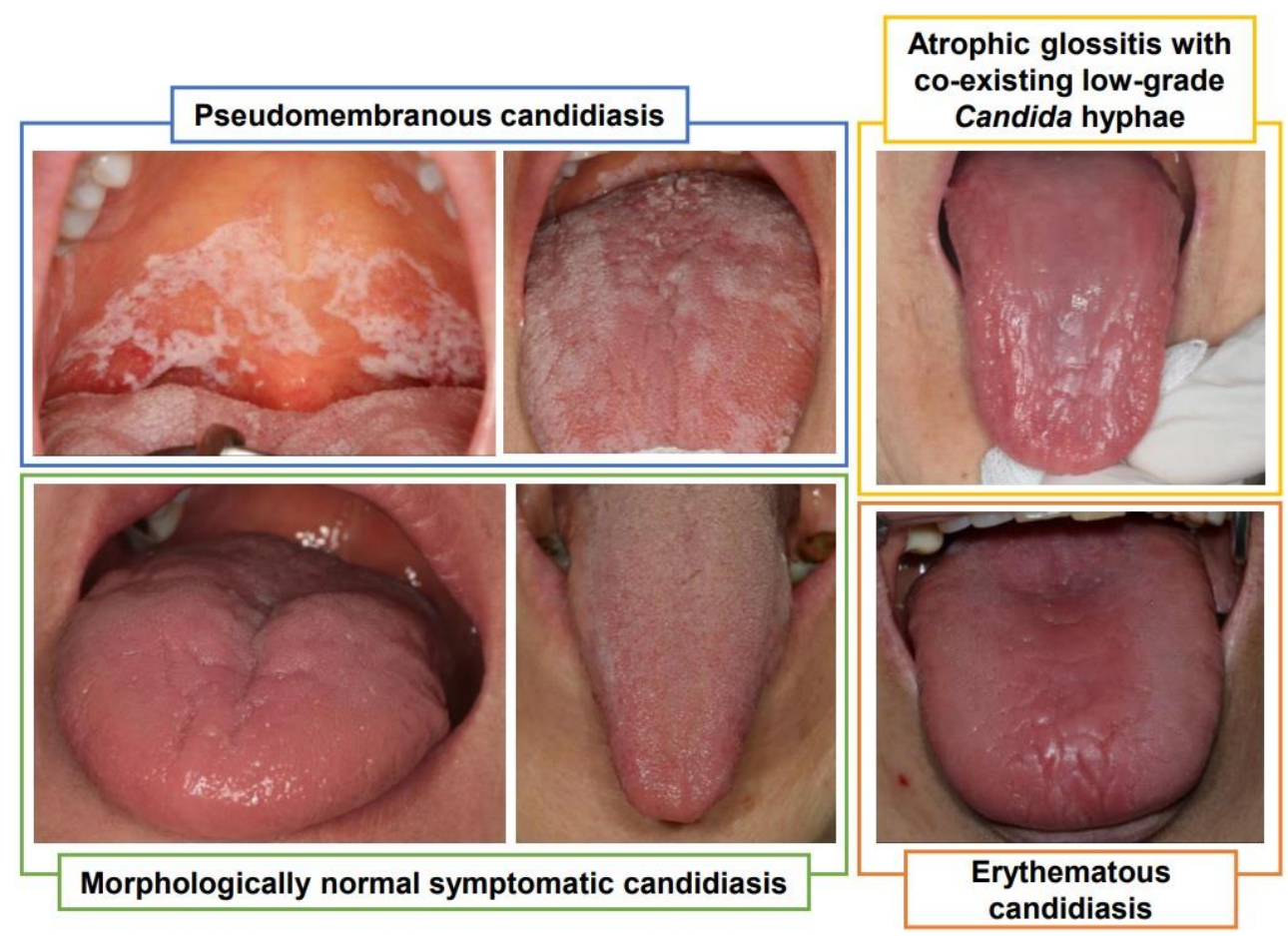

Figure 1. Subtypes of oral candidiasis based on morphologic variants.

Blue box: pseudomembranous candidiasis with diffuse and easily removable whitish curd-like pseudomembrane in the oral cavity.

Green box: morphologically normal symptomatic candidiasis presenting normal (left) or mild papillary hyperkeratosis (right) on the tongue dorsum with high-grade Candida hyphae infection. 
Yellow box: Atrophic glossitis with diffuse severe tongue papilla atrophy, low-grade Candida hyphae infection and accompanying systemic disorders (low red blood cell count, low hemoglobin level and vitamin B12 deficiency). The mucosal atrophy or moderate intensity of stimulated pain were not relieved by topical anti-fungal agent (fluconazole) use in this patient.

Orange box: erythematous candidiasis presenting partial tongue atrophy with highgrade Candida hyphae infection.

\subsection{Risk Factors of OC Subtypes}

The comparison of clinical characteristics in non-PC subtypes is described in Table 3. The proportion of moderate and severe stimulated pain cases were significantly higher in MNSC $(97.1 \%)$ than EC $(72.8 \%)(p<0.05)$. The other characteristics were statistically similar between the two groups.

Table 3. Clinical characteristics of morphologically normal symptomatic candidiasis and erythematous candidiasis.

\begin{tabular}{|c|c|c|c|}
\hline Characteristics & $\begin{array}{c}\text { Morphologically Normal } \\
\text { Symptomatic OC }\end{array}$ & Erythematous OC & $p$ Value \\
\hline \multicolumn{4}{|c|}{ Number of subjects/total (\%), unless otherwise stated } \\
\hline \multicolumn{4}{|c|}{ Age } \\
\hline$<49$ years & $5 / 35(14.3)$ & $2 / 33(6.1)$ & 0.432 \\
\hline $50-69$ years & $12 / 35(34.3)$ & $15 / 33(45.5)$ & \\
\hline$\leq 70$ years & $18 / 35(51.4)$ & $16 / 33(48.5)$ & \\
\hline \multicolumn{4}{|c|}{ Sex } \\
\hline Female $(\%)$ & $30 / 35(85.7)$ & $28 / 33(84.8)$ & 0.920 \\
\hline \multicolumn{4}{|c|}{ Pain symptoms } \\
\hline \multicolumn{4}{|c|}{ Spontaneous pain intensity } \\
\hline None & $14 / 35(40.0)$ & $16 / 33(48.5)$ & 0.417 \\
\hline Mild a & $4 / 35(11.4)$ & $7 / 33(21.2)$ & \\
\hline Moderate $^{\mathrm{a}}$ & $13 / 35(37.1)$ & $7 / 33(21.2)$ & \\
\hline Severe $^{\mathrm{a}}$ & $4 / 35(11.4)$ & $3 / 33(9.1)$ & \\
\hline \multicolumn{4}{|c|}{ Stimulated pain intensity } \\
\hline None & $0 / 35(0)$ & $4 / 33(12.1)$ & 0.039 \\
\hline Mild a & $1 / 35(2.9)$ & $5 / 33(15.2)$ & \\
\hline Moderate $^{a}$ & $16 / 35(45.7)$ & $12 / 33(36.4)$ & \\
\hline Severe ${ }^{a}$ & $18 / 35(51.4)$ & $12 / 33(36.4)$ & \\
\hline Presence of AggSt & $32 / 35(91.4)$ & $25 / 33(75.8)$ & 0.182 \\
\hline Presence of AllSt & $0 / 35(0)$ & $1 / 33(3.0)$ & \\
\hline No pain difference & $3 / 35(8.6)$ & 7/33 (21.2) & \\
\hline \multicolumn{4}{|c|}{ Initial antifungal therapy response, topical fluconazole syrup, $2 \mathrm{w}$} \\
\hline Effective on $\mathrm{SpP}^{\mathrm{b}}$ & $10 / 27(37.0)$ & $9 / 27(33.3)$ & 0.958 \\
\hline Non-effective on $\mathrm{SpP} \mathrm{b}^{\mathrm{b}}$ & $2 / 27(7.4)$ & $2 / 27(7.4)$ & \\
\hline No SpP & $15 / 27(55.6)$ & $16 / 27(59.3)$ & \\
\hline Effective on $\mathrm{StP}^{\mathrm{b}}$ & $17 / 22(77.3)$ & $16 / 21(76.2)$ & 0.285 \\
\hline Non-effective on StP $\mathrm{b}$ & $5 / 22(22.7)$ & $3 / 21(14.3)$ & \\
\hline No StP & $0 / 22(0)$ & $2 / 21(9.5)$ & \\
\hline
\end{tabular}


Table 3. Cont.

\begin{tabular}{|c|c|c|c|}
\hline Characteristics & $\begin{array}{c}\text { Morphologically Normal } \\
\text { Symptomatic OC }\end{array}$ & Erythematous OC & $p$ Value \\
\hline \multicolumn{4}{|c|}{ Accompanied conditions } \\
\hline Smoking ${ }^{\mathrm{c}}$ & $1 / 32(3.1)$ & $1 / 32(3.1)$ & 0.982 \\
\hline Alcohol ${ }^{\mathrm{c}}$ & $1 / 32(3.1)$ & $0 / 32(0)$ & 0.313 \\
\hline Denture $^{\mathrm{c}}$ & $12 / 32(37.5)$ & $10 / 29(34.5)$ & 0.806 \\
\hline Hypertension & $15 / 35(42.9)$ & $9 / 33(27.3)$ & 0.179 \\
\hline Diabetes mellitus & $7 / 35(20.0)$ & $4 / 33(12.1)$ & 0.378 \\
\hline Hepatitis B & $1 / 35(2.9)$ & $0 / 33(0)$ & 0.328 \\
\hline \multicolumn{4}{|c|}{ Salivary flow rate and presence of hyposecretion } \\
\hline Low USFR $^{\mathrm{c}}$ & $21 / 31(67.7)$ & $16 / 26(61.5)$ & 0.625 \\
\hline Low SSFR ${ }^{c}$ & $25 / 31(80.6)$ & $17 / 26(65.4)$ & 0.193 \\
\hline \multicolumn{4}{|c|}{ Complete blood counts } \\
\hline Low $\mathrm{RBC}^{\mathrm{c}}$ & $6 / 28(21.4)$ & $7 / 28(25.0)$ & 0.752 \\
\hline Low hemoglobin ${ }^{c}$ & $5 / 28(17.9)$ & $8 / 28(28.6)$ & 0.342 \\
\hline \multicolumn{4}{|c|}{ Nutrition blood levels } \\
\hline Vitamin B12 def ${ }^{c}$ & $0 / 25(0)$ & $1 / 24(4.2)$ & 0.302 \\
\hline Folate def ${ }^{\mathrm{c}}$ & $0 / 25(0)$ & $0 / 24(0)$ & $\ldots$ \\
\hline Zinc def ${ }^{c}$ & $1 / 23(4.3)$ & $3 / 24(12.5)$ & 0.317 \\
\hline Albumin def ${ }^{c}$ & $1 / 26(3.8)$ & $1 / 28(3.6)$ & 0.957 \\
\hline Serum iron def ${ }^{c}$ & $3 / 21(14.3)$ & $3 / 18$ (16.7) & 1.000 \\
\hline
\end{tabular}

$\overline{\text { Differences between proportions were determined by the chi square test and range distributions were compared }}$ by either Mann-Whitney U test or Kruskal-Wallis $H$ test. $p$ values $<0.05$ are in bold. ${ }^{\text {a }}$ Pain intensity: mild, $<$ NRS4; moderate, $\leq$ NRS4, $<$ NRS7; severe, $7 \leq$ NRS $\leq 10$. ${ }^{\text {b }}$ Effective: full or partial pain relief. ${ }^{c}$ Standard: denture, full or partial; smoking, daily; alcohol, consumption over 3 times / week; USFR, $<0.1 \mathrm{~mL} / \mathrm{min}$; SSFR $<0.7 \mathrm{~mL} / \mathrm{min} ; \mathrm{RBC}, \mathrm{F}:<3.8\left(10^{6} / \mu \mathrm{L}\right)$, M: $<4.4 ;\left(10^{6} / \mu \mathrm{L}\right)$; hemoglobin, F: $<11.7$ (g/dL), M: $<13.0$ (g/dL); vitamin B12, <211 (pg/mL); folate, <3.1 (ng/mL); zinc, <66 ( $\mathrm{gg} / \mathrm{dL})$; albumin, $<3.3(\mathrm{~g} / \mathrm{dL})$; serum iron, $<40(\mu \mathrm{g} / \mathrm{dL})$. Abbreviations: SpP, spontaneous pain; StP, stimulated pain; AggSt, pain aggravation by stimulation; AllSt, pain alleviation by stimulation; USFR, unstimulated salivary flow rate; SSFR, stimulated salivary flow rate; RBC, red blood cell count; F, female; M, male; def, deficiency; IQR, interquartile range.

Logistic regression of risk factors in MNSC and EC are detailed in Table 4. Multiple logistic regression models for non-PC subtypes revealed that low unstimulated salivary flow rate, but not stimulated salivary flow rate, was a significant risk factor in both MNSC (OR 5.3, 95\% CI 1.8-15.4, $p<0.01$ ) and EC (OR 8.2, 95\% CI 2.3-28.9, $p<0.01$ ) models. Denture was a significant risk factor only in the MNSC model (OR 4.9, 95\% CI 1.5-15.6, $p<0.01)$. Other systemic factors, such as complete blood counts and nutrition blood levels, were not risk factors of either MNSC or EC.

Table 4. Logistic regression of risk factors in morphologically normal symptomatic candidiasis and erythematous candidiasis.

\begin{tabular}{ccccc}
\hline \multirow{2}{*}{ Variates $^{\text {a }}$} & \multicolumn{2}{c}{$\begin{array}{c}\text { Morphologically Normal } \\
\text { Symptomatic OC }\end{array}$} & \multicolumn{2}{c}{ Erythematous OC } \\
\cline { 2 - 5 } & \multicolumn{2}{c}{ Simple logistic regression } & \\
\hline & $2.1(1.2-3.8)$ & $\mathbf{0 . 0 0 9}$ & $2.5(1.4-4.7)$ & $\mathbf{0 . 0 0 3}$ \\
\hline Age & $1.7(0.6-4.7)$ & 0.281 & $1.6(0.6-4.4)$ & 0.347 \\
\hline Sex & $0.4(0.1-3.4)$ & 0.418 & $0.4(0.1-3.3)$ & 0.401 \\
\hline Smoking & $0.5(0.1-3.7)$ & 0.464 & $\ldots$ & \\
\hline Alcohol & $5.3(2.3-12.5)$ & $<0.001$ & $4.7(1.9-11.5)$ & $\mathbf{0 . 0 0 1}$ \\
\hline Denture & $1.9(0.9-4.0)$ & 0.087 & $1.0(0.4-2.2)$ & 0.904 \\
\hline Hypertension & & &
\end{tabular}


Table 4. Cont.

\begin{tabular}{|c|c|c|c|c|}
\hline \multirow[t]{2}{*}{ Variates $^{a}$} & \multicolumn{2}{|c|}{$\begin{array}{l}\text { Morphologically Normal } \\
\text { Symptomatic OC }\end{array}$} & \multicolumn{2}{|c|}{ Erythematous OC } \\
\hline & Odds Ratio (95\% CI) & $p$ Value & Odds Ratio (95\% CI) & $p$ Value \\
\hline Diabetes mellitus & $1.7(0.7-4.3)$ & 0.250 & $1.0(0.3-2.9)$ & 0.928 \\
\hline Hepatitis B & $2.0(0.2-19.6)$ & 0.559 & $\ldots$ & \\
\hline Low USFR & $6.6(2.8-15.4)$ & $<0.001$ & $5.0(2.1-12.1)$ & $<0.001$ \\
\hline Low SSFR & $4.1(1.6-10.6)$ & 0.004 & $1.9(0.8-4.5)$ & 0.163 \\
\hline Low RBC & $2.1(0.7-5.6)$ & 0.163 & $2.5(1.0-6.6)$ & 0.063 \\
\hline Low hemoglobin & $2.6(0.8-7.8)$ & 0.097 & $4.7(1.8-12.6)$ & 0.002 \\
\hline Vit B12 def & $\ldots$ & & $3.6(0.3-41.1)$ & 0.305 \\
\hline Folate def & $\ldots$ & & $\ldots$ & \\
\hline Zinc def & $0.2(0.03-1.7)$ & 0.150 & $0.7(0.2-2.5)$ & 0.582 \\
\hline Albumin def & $7.1(0.4-116.8)$ & 0.171 & 6.6(0.4-107.9) & 0.188 \\
\hline Iron def & $8.4(1.6-44.7)$ & 0.013 & $8.4(1.6-44.7)$ & 0.013 \\
\hline \multicolumn{5}{|c|}{ Multiple logistic regression } \\
\hline Denture & $4.9(1.5-15.6)$ & 0.007 & $3.3(1.0-10.9)$ & 0.054 \\
\hline Low USFR & $5.3(1.8-15.4)$ & 0.002 & $8.2(2.3-28.9)$ & 0.001 \\
\hline Low SSFR & $1.8(0.5-6.0)$ & 0.342 & $0.5(0.1-1.8)$ & 0.297 \\
\hline Low hemoglobin & $2.7(0.6-12.2)$ & 0.205 & $2.5(0.6-11.6)$ & 0.231 \\
\hline AUC (IQR) & $0.8(0.7-0.9)$ & $<0.001$ & $0.8(0.7-0.9)$ & $<0.001$ \\
\hline
\end{tabular}

The $\mathrm{NH}$ (no observed hyphae) group is the reference for logistic regression analysis. $p$ value $<0.05$ are in bold. ' Standard: age, $<50,50-69, \leq 70$, years; sex, female; denture, full or partial; smoking, daily; alcohol, consumption over 3 times/week; USFR, $<0.1 \mathrm{~mL} / \mathrm{min}$; SSFR, $<0.7 \mathrm{~mL} / \mathrm{min}$; RBC, F: $<3.8\left(10^{6} / \mu \mathrm{L}\right), \mathrm{M}:<4.4$ $\left(10^{6} / \mu \mathrm{L}\right)$; hemoglobin, F: $<11.7(\mathrm{~g} / \mathrm{dL}), \mathrm{M}:<13.0(\mathrm{~g} / \mathrm{dL})$; vitamin B12, $<211(\mathrm{pg} / \mathrm{mL})$; folate, $<3.1(\mathrm{ng} / \mathrm{mL}) ;$ zinc, $<66$ ( $\mu \mathrm{g} / \mathrm{dL})$; albumin, $<3.3$ (g/dL); serum iron, $<40(\mu \mathrm{g} / \mathrm{dL})$. Abbreviations: OC, oral candidiasis; CI, confidence interval; F, female; M, male; USFR, unstimulated salivary flow rate; SSFR, stimulated salivary flow rate; def, deficiency; AUC, area under curve; IQR, interquartile range.

\section{Discussion}

The opportunistic nature of Candida infection and its various clinical phenotypes poses difficulties during clinical diagnosis and control of OC $[16,20,21]$. The formation of Candida biofilms during infection is assumed to contribute to its diverse clinical characteristics $[10,19]$. Because clinical and pathologic examinations of OC had been confirmed and widely accepted before the concept of Candida biofilms emerged, previous studies on the clinical relevance of Candida infection were mainly based on the whole Candida carriage or cultured specimens under controlled experimental conditions [18,20-24]. Among existing diagnostic methods for OC, smears are effective in collecting tongue biofilms. Candida hyphae, intermixed yeasts, keratinocytes, extracellular matrix and bacteria can be scraped from the mucosal surface onto a pathologic slide for examination [19,25-27]. Lack of sensitivity has been frequently mentioned as a major disadvantage of smears, but in our opinion, the insufficient sensitivity is due to a lack of capturing planktonic microorganisms, which are generally commensal Candida carriage $[15,20]$. Hyphae, which indicate active disease progression, attached to and infiltrate the superficial mucosa and require pressured mucosal scraping for appropriate sampling [7,8,21]. Furthermore, biofilms provide their contents with additional surface adherence, which facilitates their collection by smears [28-30]. To address the difficulty of evaluating smears quantitatively [20,31], we arranged a novel hyphae assessing method to evaluate the clinical characteristics of non-pseudomembranous OC. High/low hyphae grade discrimination not only revealed specific pain patterns of the disease, but also showed us that the clinical symptoms of Candida infection may be 
non-specific and indistinguishable from other painful or erythematous soft tissue lesions at the low hyphae grade phase.

In addition to the classic PC and EC subtypes, we characterized a previously neglected subtype of OC, provisionally termed MNSC. Although a previous study has noted a few cases of normal tongue appearance in healthy humans with observable Candida hyphae [18], this is the first study to suggest that normal tongue morphology in conjunction with pain symptoms is a definite clinical feature in OC. Since OC classification has been primarily clinical-morphology-based, this subtype has gone unheeded in the clinic and may be misdiagnosed as other painful oral diseases (e.g., burning mouth syndrome) with coexisting commensal Candida $[16,17,32,33]$. Contrary to conventional classification criteria, we believe that specific pain symptoms and hyphae grade are the major diagnostic factors for OC, rather than mucosal morphology. Although a previous study reported painful OC without external abnormalities, the evaluation had been based on the whole Candida carriage without discrimination of the disease phenotype, hyphae [34].

Candida-induced pain has been ambiguously described in the literature, such as burning, sensitive or painful, mostly without clear distinction between SpP and StP $[16,20,35]$. The association of StP and Candida carriage has been noticed in previous studies, but the correlation between StP and active Candida hyphae infection or soft tissue Candida biofilms has not been examined before $[33,34]$. Our findings indicated moderate to severe StP as a specific pain characteristic of Candida hyphae infection in the tongue biofilm. Moreover, $\mathrm{SpP}$ or AllSt, which are known features of burning mouth syndrome or atrophic glossitis caused by nutritional deficiencies, did not show association with high-grade Candida hyphae infection [36-39]. Consistent with our results, other studies have reported antifungal treatment to be ineffective in patients with mainly SpP [33,34]. Altogether, these suggest that concrete discrimination of pain features is informative for clinical differentiation of OC. Interestingly, we observed that MNSC had a more advanced degree of StP intensity than EC. Moreover, there were no painless patients in MNSC, unlike EC. This implied that mucosal atrophy or erythema may not be the major cause of Candida-induced pain.

Factors associated with morphologic phenotype diversity are poorly understood in OC. As specific pain symptoms are universal in non-pseudomembranous OC, we further investigated factors associated with clinical morphology in Candida infection. Conspicuous hyphae transition and proliferation have been observed in all PC, EC and MNSC, although the degree of direct hyphae/keratinocyte infiltration may be relatively indistinct in EC [21,37,40,41]. Candida hyphae are known to induce increased keratin differentiation and pseudomembrane formation $[3,21,40]$, but whether they are responsible for contrasting clinical features of mucosal atrophy or normal differentiation is an intricate issue and rather a paradox. Despite that the presence of tongue atrophy and pain may suggest Candida infection, atrophic glossitis can arise from other conditions including salivary hyposecretion, anemia and nutritional deficiencies [17,35-39]. Possible local and systemic risk factors of morphological non-PC subtypes did not differ except for denture use. Although denture use was not statistically significant in EC, it showed a strong tendency as a risk factor as well. These results indicated that co-existing anemia or nutritional deficiencies had minimal impact on atrophic or erythematous morphology in definite OC with high-grade hyphae.

Other than clinical factors, the contents of Candida biofilm may contribute to OC morphology phenotypes. Although our retrospective study focused mainly on the clinical relevance of the hyphae content within the smeared tongue material, tongue smears can provide a comprehensive profile of non-hyphae contents in the biofilm. The composition of extracellular matrix, immune cells and bacteria and their interactions with Candida organisms within the biofilm may influence the differentiation of underlying epithelial cells $[11,27,29,42,43]$. Further study of non-Candida biofilm contents may lead to systemic insights into the clinical relevance of Candida infection.

Various local or systemic conditions have been suggested as causative or predisposing factors of OC [40-43]. These factors may directly affect fungal microorganisms or may induce an immunosuppressive state or biofilm formation that can enhance Candida 
pathogenesis [42,44-48]. In general, many patients have more than one possible risk factor for OC, and the degree of the risk conditions vary among individuals. Furthermore, the association between Candida and possible risk factors can differ based on the method used for fungus measurement, as Candida has a dimorphic presence.

Candida infection related with denture use is called denture stomatitis. It typically arises on the palate mucosa in contact with the acrylic surface of maxillary dentures [48-51]. Candida-induced denture stomatitis is presented as red, atrophic and sore mucosa at the interface of the denture surface and soft tissue. Co-associated bacteria species have been suggested to aid Candida biofilm formation in dentures [52-57]. Dentures were significantly involved with MNSC showing normal tongue mucosa in this study. Although erythema and atrophy are characteristic in denture stomatitis, they do not seem to be significant in the tongue mucosa of denture users. The tongue does not have a tight and intimate contact with the denture surface as seen in the palate or gingiva, thus the association between dentures and the tongue Candida biofilm is suspected to be indirect. Dentures may contribute as a reservoir for increased oral Candida carriage that can lead to Candida hyphae and biofilm formation at the tongue in appropriate conditions.

Hyposalivation is one of the most mentioned risk factors of OC. Candida carriages have been commonly observed in xerostomia [52,56,57]. Saliva contains antifungal proteins and antibodies that inhibit Candida adhesion and colonization and stimulates an innate immune response against Candida microorganisms [52,57,58]. We identified that reduced unstimulated saliva, but not stimulated saliva, was a common risk factor in MNSC and EC multiple regression models. Other studies observed that Candida count had an inverse relation with both stimulated and unstimulated salivary flow, but the investigations were based on planktonic fungal carriage collected from saliva [24,48,49,58]. Reduced stimulated and unstimulated saliva have different molecule compositions [59], but their pathogenic effect on Candida colonization and biofilm formation are not known.

Smoking, alcohol consumption, hypertension, diabetes mellitus and hepatitis B were not risk factors for non-PC subtypes in this study. Chemicals in cigarette smoke has been proposed to induce Candida infection and biofilm formation in vitro, but its affects in clinical settings are controversial [18,60-62]. Candida has been suspected to be related to diabetes in both adults and children [63-67]. In contrast to our results, Bartholomew et al. observed that invasive Candida was more frequently identified in diabetic patients than non-diabetic subjects by oral cytologic smears [64]. Sato et al. reported that hypertension and daily alcohol intake were associated with greater carriage of Candida albicans in the elderly resided at the post-disaster region, but this was not seen in our results based on invasive Candida [68].

\section{Conclusions}

New insights on Candida biofilm formation during hyphae transition and infiltration have brought up the need for clinical assessment based on invasive hyphae within the biofilm. In this study, we evaluated the clinical relevance of Candida infection in smeared tongue biofilms based on our novel hyphae grade method. We discovered that stimulated pain was a critical and specific characteristic, while tongue mucosal atrophy was a significant but not a specific characteristic in non-PC subtypes. Importantly, we report a morphologically normal, yet symptomatic, OC subtype named "morphologically normal symptomatic candidiasis" as a common non-PC subtype. The morphological differences of MNSC and EC were thought to be minimally affected by clinical risk factors; instead, other factors such as non-fungal components of the Candida biofilm may contribute to the diversity of morphologic phenotypes in non-PC subtypes.

Supplementary Materials: The following are available online at https:/ /www.mdpi.com/2309-608 X/7/2/77/s1, Figure S1: Preliminary data of clinical characteristic distribution by total hyphae value, Table S1: Sensitivity, specificity and accuracy of hyphae value candidates on preliminary data. 
Author Contributions: Conceptualization, E.C., J.-S.K., H.-J.A.; methodology, E.C., Y.P., K.-Y.K.; validation, H.S.K., K.-Y.K.; investigation, E.C., Y.P.; data curation, E.C., Y.P., D.H., H.S.K.; writingoriginal draft preparation, E.C., Y.P.; writing—review and editing, J.-S.K., H.-J.A.; supervision, J.-S.K., H.-J.A.; funding acquisition, K.-Y.K., H.-J.A. All authors have read and agreed to the published version of the manuscript.

Funding: This work was supported by the National Research Foundation of Korea (NRF) grant funded by the Korea government (MSIP) (NRF-2016R1A5A2008630, NRF-2019R1A2C1003028) and the BK21 FOUR Project, Yonsei University College of Dentistry.

Institutional Review Board Statement: The study was conducted according to the guidelines of the Declaration of Helsinki and approved by the Institutional Review Board of Yonsei University Dental Hospital (2-2017-0001).

Informed Consent Statement: Patient consent was waived due to its minimal risk to the subjects and that all the retrospective information had been obtained during clinical procedures regardless of research purpose.

Data Availability Statement: The data presented in this study are available on request from the corresponding author. The data are not publicly available due to the privacy issues.

Acknowledgments: We thank Eph Tunkle for editing and proofreading of the manuscript.

Conflicts of Interest: The authors declare no conflict of interest. The funders had no role in the design of the study; in the collection, analyses, or interpretation of data; in the writing of the manuscript, or in the decision to publish the results.

\section{References}

1. Thompson, D.S.; Carlisle, P.L.; Kadosh, D. Coevolution of morphology and virulence in Candida species. Eukaryot Cell 2011, 10, 1173-1182. [CrossRef] [PubMed]

2. Sudbery, P.E. Growth of Candida albicans hyphae. Nat. Rev. Microbiol. 2011, 9, 737-748. [CrossRef] [PubMed]

3. Rollenhagen, C.; Wöllert, T.; Langford, G.M.; Sundstrom, P. Stimulation of cell motility and expression of late markers of differentiation in human oral keratinocytes by Candida albicans. Cell Microbiol. 2009, 11, 946-966. [CrossRef] [PubMed]

4. Ene, I.V.; Bennett, R.J. Hwp1 and related adhesins contribute to both mating and biofilm formation in Candida albicans. Eukaryot Cell 2009, 8, 1909-1913. [CrossRef]

5. Nobile, C.J.; Mitchell, A.P. Genetics and genomics of Candida albicans biofilm formation. Cell Microbiol. 2006, 8, $1382-1391$. [CrossRef]

6. Ramage, G.; VandeWalle, K.; López-Ribot, J.L.; Wickes, B.L. The filamentation pathway controlled by the Efg1 regulator protein is required for normal biofilm formation and development in Candida albicans. FEMS Microbiol. Lett. 2002, 214, 95-100. [CrossRef]

7. Yang, W.; Yan, L.; Wu, C.; Zhao, X.; Tang, J. Fungal invasion of epithelial cells. Microbiol. Res. 2014, 169, 803-810. [CrossRef]

8. Richardson, J.P.; Ho, J.; Naglik, J.R. Candida-epithelial interactions. J. Fungi 2018, 4, 22. [CrossRef]

9. Lo, H.-J.; Köhler, J.R.; DiDomenico, B.; Loebenberg, D.; Cacciapuoti, A.; Fink, G.R. Nonfilamentous C. albicans mutants are avirulent. Cell 1997, 90, 939-949. [CrossRef]

10. Dongari-Bagtzoglou, A.; Kashleva, H.; Dwivedi, P.; Diaz, P.; Vasilakos, J. Characterization of mucosal Candida albicans biofilms. PLoS ONE 2009, 4, e7967. [CrossRef]

11. O’Donnell, L.E.; Millhouse, E.; Sherry, L.; Kean, R.; Malcolm, J.; Nile, C.J.; Ramage, G. Polymicrobial Candida biofilms: Friends and foe in the oral cavity. FEMS Yeast Res. 2015, 15. [CrossRef] [PubMed]

12. Ganguly, S.; Mitchell, A.P. Mucosal biofilms of Candida albicans. Curr. Opin. Microbiol. 2011, 14, 380-385. [CrossRef]

13. Cavalheiro, M.; Teixeira, M.C. Candida biofilms: Threats, challenges, and promising strategies. Front. Med. 2018, 5, 28. [CrossRef] [PubMed]

14. Wu, X.; Zhang, S.; Li, H.; Shen, L.; Dong, C.; Sun, Y.; Chen, H.; Xu, B.; Zhuang, W.; Deighton, M.; et al. Biofilm formation of Candida albicans facilitates fungal infiltration and persister cell formation in vaginal candidiasis. Front. Microbiol. $2020,11$. [CrossRef] [PubMed]

15. Hawser, S.P.; Douglas, L.J. Biofilm formation by Candida species on the surface of catheter materials in vitro. Infect. Immun. 1994, 62, 915-921. [CrossRef]

16. Lynch, D.P. Oral candidiasis. History, classification, and clinical presentation. Oral Surg. Oral Med. Oral Pathol. 1994, 78, 189-193. [CrossRef]

17. Holmstrup, P.; Axéll, T. Classification and clinical manifestations of oral yeast infections. Acta Odontol. Scand. 1990, 48, 57-59. [CrossRef]

18. Arendorf, T.M.; Walker, D.M. The prevalence and intra-oral distribution of Candida albicans in man. Arch. Oral Biol. 1980, 25, 1-10. [CrossRef] 
19. Harriott, M.M.; Noverr, M.C. Importance of Candida-bacterial polymicrobial biofilms in disease. Trends Microbiol. 2011, 19, 557-563. [CrossRef]

20. Williams, D.W.; Lewis, M.A. Isolation and identification of Candida from the oral cavity. Oral Dis. 2000, 6, 3-11. [CrossRef]

21. Tooyama, H.; Matsumoto, T.; Hayashi, K.; Kurashina, K.; Kurita, H.; Uchida, M.; Kasuga, E.; Honda, T. Candida concentrations determined following concentrated oral rinse culture reflect clinical oral signs. BMC Oral Health 2015, 15, 150. [CrossRef]

22. White, P.L.; Williams, D.W.; Kuriyama, T.; Samad, S.A.; Lewis, M.A.O.; Barnes, R.A. Detection of Candida in concentrated oral rinse cultures by real-time PCR. J. Clin. Microbiol. 2004, 42, 2101-2107. [CrossRef]

23. Epstein, J.B.; Pearsall, N.N.; Truelove, E.L. Quantitative relationships between Candida albicans in saliva and the clinical status of human subjects. J. Clin. Microbiol. 1980, 12, 475-476. [CrossRef]

24. Torres, S.R.; Peixoto, C.B.; Caldas, D.M.; Silva, E.B.; Magalhães, F.A.; Uzeda, M.; Nucci, M. Clinical aspects of Candida species carriage in saliva of xerotomic subjects. Med. Mycol. 2003, 41, 411-415. [CrossRef]

25. Rodrigues, M.E.; Gomes, F.; Rodrigues, C.F. Candida spp./bacteria mixed biofilms. J. Fungi 2019, 6, 5. [CrossRef]

26. Chandra, J.; Kuhn, D.M.; Mukherjee, P.K.; Hoyer, L.L.; McCormick, T.; Ghannoum, M.A. Biofilm formation by the fungal pathogen Candida albicans: Development, architecture, and drug resistance. J. Bacteriol. 2001, 183, 5385-5394. [CrossRef]

27. Hawser, S.P.; Baillie, G.S.; Douglas, L.J. Production of extracellular matrix by Candida albicans biofilms. J. Med. Microbiol. 1998, 47, 253-256. [CrossRef]

28. Nobile, C.J.; Schneider, H.A.; Nett, J.E.; Sheppard, D.C.; Filler, S.G.; Andes, D.R.; Mitchell, A.P. Complementary adhesin function in C. albicans biofilm formation. Curr. Biol. 2008, 18, 1017-1024. [CrossRef]

29. Soll, D.R. Candida biofilms: Is adhesion sexy? Curr. Biol. 2008, 18, R717-R720. [CrossRef]

30. McCall, A.D.; Pathirana, R.U.; Prabhakar, A.; Cullen, P.J.; Edgerton, M. Candida albicans biofilm development is governed by cooperative attachment and adhesion maintenance proteins. NPJ Biofilms Microbiomes 2019, 5, 21. [CrossRef]

31. Byadarahally Raju, S.; Rajappa, S. Isolation and identification of Candida from the oral cavity. ISRN Dent. 2011, $2011,487921$. [CrossRef] [PubMed]

32. Osaki, T.; Yoneda, K.; Yamamoto, T.; Ueta, E.; Kimura, T. Candidiasis may induce glossodynia without objective manifestation. Am. J. Med. Sci. 2000, 319, 100-105. [CrossRef]

33. Terai, H.; Shimahara, M. Tongue pain: Burning mouth syndrome vs. Candida- associated lesion. Oral Dis. 2007, 13, 440-442. [CrossRef]

34. Terai, H.; Shimahara, M. Glossodynia from Candida-associated lesions, burning mouth syndrome, or mixed causes. Pain Med. 2010, 11, 856-860. [CrossRef] [PubMed]

35. Sun, A.; Lin, H.P.; Wang, Y.P.; Chiang, C.P. Significant association of deficiency of hemoglobin, iron and vitamin B12, high homocysteine level, and gastric parietal cell antibody positivity with atrophic glossitis. J. Oral Pathol. Med. 2012, 41, 500-504. [CrossRef] [PubMed]

36. Demir, N.; Dogan, M.; Koc, A.; Kaba, S.; Bulan, K.; Ozkol, H.U.; Dogan, S.Z. Dermatological findings of vitamin B12 deficiency and resolving time of these symptoms. Cutan. Ocul. Toxicol. 2014, 33, 70-73. [CrossRef]

37. Bao, Z.X.; Yang, X.W.; Shi, J.; Liu, L.X. Serum zinc levels in 368 patients with oral mucosal diseases: A preliminary study. Med. Oral Patol. Oral Cir. Bucal 2016, 21, e335-e340. [CrossRef]

38. Lu, S.Y. Perception of iron deficiency from oral mucosa alterations that show a high prevalence of Candida infection. J. Formos Med. Assoc. 2016, 115, 619-627. [CrossRef]

39. Wu, Y.-C.; Wang, Y.-P.; Chang, J.Y.-F.; Cheng, S.-J.; Chen, H.-M.; Sun, A. Oral manifestations and blood profile in patients with iron deficiency anemia. J. Formos Med. Assoc. 2014, 113, 83-87. [CrossRef]

40. Singh, A.; Verma, R.; Murari, A.; Agrawal, A. Oral candidiasis: An overview. J. Oral Maxillofac. Pathol. 2014, 18, S81-S85. [CrossRef]

41. Martins, N.; Ferreira, I.C.; Barros, L.; Silva, S.; Henriques, M. Candidiasis: Predisposing factors, prevention, diagnosis and alternative treatment. Mycopathologia 2014, 177, 223-240. [CrossRef]

42. Cannon, R.D.; Holmes, A.R.; Mason, A.B.; Monk, B.C. Oral Candida: Clearance, colonization, or candidiasis? J. Dent. Res. 1995, 74, 1152-1161. [CrossRef]

43. Vila, T.; Sultan, A.S.; Montelongo-Jauregui, D.; Jabra-Rizk, M.A. Oral candidiasis: A disease of opportunity. J. Fungi 2020, 6, 15. [CrossRef]

44. Netea, M.G.; Joosten, L.A.B.; van der Meer, J.W.M.; Kullberg, B.-J.; van de Veerdonk, F.L. Immune defence against Candida fungal infections. Nat. Rev. Immunol. 2015, 15, 630-642. [CrossRef]

45. Fidel, P.L., Jr. Immunity to Candida. Oral Dis. 2002, 8 (Suppl. 2), 69-75. [CrossRef] [PubMed]

46. Eix, E.F.; Nett, J.E. How biofilm growth affects Candida-host interactions. Front Microbiol. 2020, 11, 1437. [CrossRef] [PubMed]

47. Heimdahl, A.; Nord, C.E. Oral yeast infections in immunocompromised and seriously diseased patients. Acta Odontol. Scand. 1990, 48, 77-84. [CrossRef]

48. Nadig, S.D.; Ashwathappa, D.T.; Manjunath, M.; Krishna, S.; Annaji, A.G.; Shivaprakash, P.K. A relationship between salivary flow rates and Candida counts in patients with xerostomia. J. Oral Maxillofac. Pathol. 2017, 21, 316. [CrossRef]

49. Torres, S.R.; Peixoto, C.B.; Caldas, D.M.; Silva, E.B.; Akiti, T.; Nucci, M.; de Uzeda, M. Relationship between salivary flow rates and Candida counts in subjects with xerostomia. Oral Surg. Oral Med. Oral Pathol. Oral Radiol. Endod. 2002, 93, 149-154. [CrossRef] 
50. Karbach, J.; Walter, C.; Al-Nawas, B. Evaluation of saliva flow rates, Candida colonization and susceptibility of Candida strains after head and neck radiation. Clin. Oral Investig. 2012, 16, 1305-1312. [CrossRef]

51. Buranarom, N.; Komin, O.; Matangkasombut, O. Hyposalivation, oral health, and Candida colonization in independent dentate elders. PLoS ONE 2020, 15, e0242832. [CrossRef]

52. Kanaguchi, N.; Narisawa, N.; Ito, T.; Kinoshita, Y.; Kusumoto, Y.; Shinozuka, O.; Senpuku, H. Effects of salivary protein flow and indigenous microorganisms on initial colonization of Candida albicans in an in vivo model. BMC Oral Health 2012, 12, 36. [CrossRef] [PubMed]

53. Khan, S.A.; Fidel, P.L., Jr.; Thunayyan, A.A.; Varlotta, S.; Meiller, T.F.; Jabra-Rizk, M.A. Impaired Histatin-5 Levels and Salivary Antimicrobial Activity against C. albicans in HIV Infected Individuals. J. AIDS Clin. Res. 2013, 4. [CrossRef] [PubMed]

54. Salvatori, O.; Puri, S.; Tati, S.; Edgerton, M. Innate immunity and saliva in Candida albicans-mediated oral diseases. J. Dent. Res. 2016, 95, 365-371. [CrossRef]

55. Linden, S.K.; Sutton, P.; Karlsson, N.G.; Korolik, V.; McGuckin, M.A. Mucins in the mucosal barrier to infection. Mucosal Immunol. 2008, 1, 183-197. [CrossRef] [PubMed]

56. Vila, T.; Rizk, A.M.; Sultan, A.S.; Jabra-Rizk, M.A. The power of saliva: Antimicrobial and beyond. PLoS Pathog. 2019, 15, e1008058. [CrossRef] [PubMed]

57. Lynge Pedersen, A.M.; Belstrøm, D. The role of natural salivary defences in maintaining a healthy oral microbiota. J. Dent. 2019, 80, S3-S12. [CrossRef] [PubMed]

58. Navazesh, M.; Wood, G.J.; Brightman, V.J. Relationship between salivary flow rates and Candida albicans counts. Oral Surg. Oral Med. Oral Pathol. Oral Radiol. Endod. 1995, 80, 284-288. [CrossRef]

59. Inui, T.; Palmer, R.J.; Shah, N.; Li, W.; Cisar, J.O.; Wu, C.D. Effect of mechanically stimulated saliva on initial human dental biofilm formation. Sci. Rep. 2019, 9, 11805. [CrossRef]

60. Soysa, N.S.; Ellepola, A.N. The impact of cigarette/tobacco smoking on oral candidosis: An overview. Oral Dis. 2005, 11, 268-273. [CrossRef]

61. Darwazeh, A.M.; Al-Dwairi, Z.N.; Al-Zwairi, A.A. The relationship between tobacco smoking and oral colonization with Candida species. J. Contemp. Dent. Prac. 2010, 11, 017-024.

62. Muzurović, S.; Hukić, M.; Babajić, E.; Smajić, R. The relationship between cigarette smoking and oral colonization with Candida species in healthy adult subjects. Med. Glas. Off. Publ. Med. Assoc. Zenica Doboj Canton Bosnia Herzeg. 2013, 10, $397-399$.

63. Olczak-Kowalczyk, D.; Pyrżak, B.; Dąbkowska, M.; Pańczyk-Tomaszewska, M.; Miszkurka, G.; Rogozińska, I.; Swoboda-Kopeć, E.; Gozdowski, D.; Kalińska, A.; Piróg, A.; et al. Candida spp. and gingivitis in children with nephrotic syndrome or type 1 diabetes. BMC Oral Health 2015, 15, 57. [CrossRef]

64. Bartholomew, G.A.; Rodu, B.; Bell, D.S. Oral candidiasis in patients with diabetes mellitus: A thorough analysis. Diabetes Care 1987, 10, 607-612. [CrossRef]

65. Mohammadi, F.; Javaheri, M.R.; Nekoeian, S.; Dehghan, P. Identification of Candida species in the oral cavity of diabetic patients. Curr. Med. Mycol. 2016, 2, 1-7. [CrossRef]

66. Chouhan, S.; Kallianpur, S.; Prabhu, K.T.; Tijare, M.; Kasetty, S.; Gupta, S. Candidal prevalence in diabetics and its species identification. Int. J. Appl. Basic Med. Res. 2019, 9, 49-54. [CrossRef]

67. Sampath, A.; Weerasekera, M.; Dilhari, A.; Gunasekara, C.; Bulugahapitiya, U.; Fernando, N.; Samaranayake, L. Type 2 diabetes mellitus and oral Candida colonization: Analysis of risk factors in a Sri Lankan cohort. Acta Odontol. Scand. 2019, 77, 508-516. [CrossRef]

68. Sato, T.; Kishi, M.; Suda, M.; Sakata, K.; Shimoda, H.; Miura, H.; Ogawa, A.; Kobayashi, S. Prevalence of Candida albicans and non-albicans on the tongue dorsa of elderly people living in a post-disaster area: A cross-sectional survey. BMC Oral Health 2017, 17, 51. [CrossRef] 